\title{
A rapidly growing human papillomavirus-positive oral tongue squamous cell carcinoma in a 21-year old female: A case report
}

\author{
EBENEZER O. APPAH ${ }^{1}$, BILLY RAY BALLARD ${ }^{2}$, MICHAEL G. IZBAN $^{2}$, CASSANDRA JOLIN $^{3}$, \\ PHILIP E. LAMMERS ${ }^{1}$, DWIGHT D. PARRISH JR ${ }^{2}$ and DANA R. MARSHALL ${ }^{2}$ \\ Departments of ${ }^{1}$ Internal Medicine, ${ }^{2}$ Pathology, Anatomy and Cell Biology and \\ ${ }^{3}$ Family and Community Medicine, Meharry Medical College, Nashville, TN 37208, USA
}

Received December 22, 2016; Accepted August 1, 2017

DOI: $10.3892 / \mathrm{ol} .2018 .8339$

\begin{abstract}
Oral tongue squamous cell carcinoma (OTSCC) has a median age at diagnosis of 62 years. The incidence of OTSCC in young adults has been increasing, and the reason is unclear. The present study describes a case, and molecular analysis, of OTSCC in a 21-year-old female. Clinical and pathological information were collected from medical records. Formalin-fixed paraffin-embedded biopsy tissue from the patient was reassessed using standard hematoxylin \& eosin staining, and immunohistochemistry was used to assess the expression of cellular p16, MutL homolog (MLH)1, MLH2, MutS homolog 6 (MSH6) and PMS1 homolog 2 (PMS2). The human papilloma virus (HPV) genome was detected by PCR analysis of the extracted DNA. The young age of the patient with OTSCC was unusual. The original pathology report indicated koilocytotic atypia, a cellular abnormality associated with HPV. Although HPV-positive oral cancer tends to occur in 'younger' individuals, 21 years is unusual. The confirmation of biologically active HPV in the tumor was obtained via the observation of strong positive staining for cellular p16. The patient described a maternal family cluster of rare cancer types, thus the possibility that this rapidly growing cancer resulted from HPV infection combined with an underlying genetic mutation causing decreased DNA-mismatch repair was explored. However, MSH1, MSH2, MSH6 and PSM2, proteins that are associated with Lynch Syndrome, were expressed at normal levels. A rapidly growing OTSCC of a 21-year-old female was determined to be HPV-positive. The patient underwent combination chemotherapy and radiation and has experienced long-term survival without recurrence. The reason this tumor grew so quickly in such a young individual remains unknown. These types of cases warrant additional
\end{abstract}

Correspondence to: Dr Dana R. Marshall, Department of Pathology, Anatomy and Cell Biology, Meharry Medical College, 1005 Dr. DB Todd Jr. Boulevard, Nashville, TN 37208, USA

E-mail: dmarshall@mmc.edu

Key words: oral tongue, mobile tongue, anterior tongue, squamous cell carcinoma, human papillomavirus, tongue cancer genomic and proteomic studies to improve understanding of this phenomenon.

\section{Introduction}

The overall incidence of oral cavity cancer in general is decreasing (1). Nevertheless, previous studies have revealed the incidence of oral tongue squamous cell carcinoma (OTSCC) is increasing, particularly in young adults $<45$ years of age (1-3). Patterns in cancer epidemiology from 2000-2013 published by the Surveillance, Epidemiology, and End Results Program 18 identified $\sim 6,520$ cases of tongue cancer, with an incidence rate that increased from 2.6-3.3 per 100,000 (4).

The role of tobacco use and alcohol consumption, traditional risk factors in the development of OTSCC, is well established. However, exposure to such risk factors has not been extensive, or may even be absent, in these young adults as compared with in older individuals. Llewellyn et al (5) identified that even in patients $>30$ years old who smoked or consumed alcohol, the duration of exposure was insufficient for the development of malignancy (5). Human papillomavirus (HPV) infection has emerged as a potential risk factor for the development of OTSCC; however, the results from prior studies are inconsistent with regard to its potential role.

The etiology of HPV-associated head and neck SCC (HNSCC) has been investigated in numerous epidemiologic studies, and the subtypes HPV16 and HPV18 have been identified as the causative agents (5). Patients with HPV-positive HNSCC tend to be younger and have minimal tobacco and alcohol use. HPV-positive HNSCC is most common in the oropharynx, particularly in the tonsils and the base of the tongue, and is usually well-differentiated. Anaya-Saavedra et al (6) revealed that high-risk HPV (HPV16 and HPV18) is strongly associated with oral cancer, in their study of oral cancer risk factors in Mexican patients (6). Conversely, other studies observed that OTSCC in young Caucasian females is characteristically negative for HPV. Possible causal factors may include genetic abnormalities, such as Fanconi anemia, other oncogenic viral infections and/or other environmental exposures, or may be associated with HPV types other than HPV16 or $18(7,8)$. This was based on the data that the infection rate of the oral cavity and tongue is low, that the incidence of HPV-associated cancer is decreasing in females and that the increasing incidence 
of OTSCC in young Caucasian females may not be due to HPV (6).

Patients with HPV-associated OTSCC have been demonstrated to exhibit a better prognosis compared with patients with non-HPV-associated OTSCC. Marur et al (9) concluded that questions associated with the role of HPV, and the natural history of oral HPV infection, remain. The result is that further studies are required for the understanding of disease progression in order to optimize the clinical management of patients with HNSCC with HPV positive disease. With this background, the etiology, risk factors and pathophysiology of OTSCC remain unknown.

The present study describes a case of OTSCC in a 21-year-old Caucasian female with insignificant conventional risk factor exposure and atypical presentation.

\section{Materials and methods}

Ethical statement. The present retrospective study was approved by the Meharry Medical College Institutional Review Board and the Medical Executive Committee at Nashville General Hospital at Meharry (Nashville, TN, USA).

Case report. A 21-year-old Caucasian female presented to the emergency room of a city safety-net hospital with a one-week history of a rapidly growing painful mass on the right side of the tongue. The patient had initially noticed it as a 'blister', which ruptured and ulcerated. The patient denied any constitutional symptoms. The patient did not report a history of radiation exposure and had no significant medical history. The patient reported malignancies in multiple relatives, including ovarian cancer (mother), brain cancer (uncle), cholangiocarcinoma (maternal grandmother), lung/laryngeal cancer (paternal grandfather) and unknown cancer types in four uncles. The patient denied any history of smoking, alcohol consumption or illicit drug use. Examination revealed an exophytic tender ulcer $3-4 \mathrm{~cm}$ in size on the right lateral region of the tongue. The patient exhibited a palpable right submandibular lymph node enlargement.

A fine needle aspiration of the right jugulodigastric lymph node revealed poorly differentiated squamous cell carcinoma. The patient underwent a panendoscopy and examination under anesthesia that indicated a $2.5 \mathrm{~cm}$ ulcerated and indurated lesion of the right lateral region of the tongue. An incisional biopsy revealed a well-moderately differentiated invasive SCC, characterized by sheets of large cells with pleomorphic vesicular nuclei, sparse eosinophilic cytoplasm, with individual cell keratinization and focal keratin pearl formation, and numerus mitotic figures (Fig. 1A). Focal clusters of malignant cells demonstrated koilocytotic atypia characterized by cytoplasmic vacuolization with thickening of the cytoplasmic membrane and mild variation in the size and shape of the nuclei (Fig. 1B). A neck computed tomography scan indicated a right lateral tongue mass with irregular enhancement in the right parapharyngeal region, and magnetic resonance imaging (MRI) demonstrated a $1.2 \times 1.8 \times 1.3 \mathrm{~cm}$ mass at the intersection of the mid and posterior third of the right tongue without crossing the raphe of the tongue. The enlarged lymph nodes involved levels IB, II and III. Chest radiography indicated no abnormalities and the brain MRI was negative for metastatic disease. Therefore, the final staging was stage III (T3N1M0) based on the 6th edition of the International Union Against Cancer (UICC) and the American Joint Committee on Cancer (AJCC) (10).

The patient received neoadjuvant chemotherapy with weekly carboplatin and paclitaxel. Following ten cycles, there was significant clinical and radiological evidence of regression in the size of the primary tumor and the lymph node. The patient then underwent a partial right glossectomy with supraomohyoid neck dissection. Histopathology indicated foci of well-poorly differentiated SCC with marked degeneration and foreign body granuloma. The neck dissection specimen demonstrated a small focus of metastatic SCC in one of nine lymph nodes. Post-operatively, the patient received chemotherapy with carboplatin and paclitaxel and concurrent radiation for nine weeks. Radiotherapy was complicated by dysphagia, for which the patient required a percutaneous gastrostomy tube. Follow-up imaging and endoscopic examination indicated no evidence of recurrence over the next three years.

The patient was eventually lost to follow-up, but returned to the oncology clinic fifteen years post-surgery. At this visit, the patient exhibited mild hypothyroidism, but no other significant long-term toxicities from the treatment and was disease free.

Koilocytotic atypia in an SCC of the head and neck has been considered to be pathognomonic for HPV. At the time of diagnosis, it was not standard practice to confirm the presence of HPV in OTSCC, so that was not performed. The presence of multiple relatively rare cancer types in the relatives, who were also fairly young, was noteworthy. The young patient age and the aggressiveness of the tongue cancer led to additional exploration of the possibility of an unusually aggressive HPV strain and/or HPV in the background of an underlying genetic condition, potentially Lynch syndrome. The presence of actively replicating HPV in the tumor was explored via immunohistochemical staining for cellular p16, which is frequently elevated in HPV-positive SCC and is the clinical lab surrogate marker for HPV.

In light of the relatively low incidence of certain Lynch Syndrome-associated malignancies in the general population, including endometrial, ovarian, gall bladder and brain cancer, a number of genetic pedigrees have clearly indicated that indexed patients are at risk (11). Based on the history provided by the patient, immunohistochemical staining was performed for four proteins, of which at least one is typically underexpressed in individuals with Lynch Syndrome. These four proteins are all involved in DNA mismatch repair and are MutL homolog (MLH) 1, MLH2, MutS homolog 6 (MSH6) and PMS1 homolog 2 (PMS2).

\section{Experimental methods}

Cellular p16 immunohistochemistry (surrogate marker for $H P V)$. Tumor tissue blocks were retrieved $\sim 12$ years post-surgery and sectioned for confirmation of tumor grade and cellular p16 staining. The resulting slides were deparaffinized using the standard xylene/ethanol/PBS protocol. Antigen retrieval was performed for $20 \mathrm{~min}$ at $98^{\circ} \mathrm{C}\left(60^{\circ} \mathrm{C}\right.$ preheat $/ 70^{\circ} \mathrm{C}$ cool down) using the LabVision ${ }^{\mathrm{TM}} \mathrm{PT}$ module and PT Module Tris pH 9 Buffer (Thermo Fisher Scientific, 


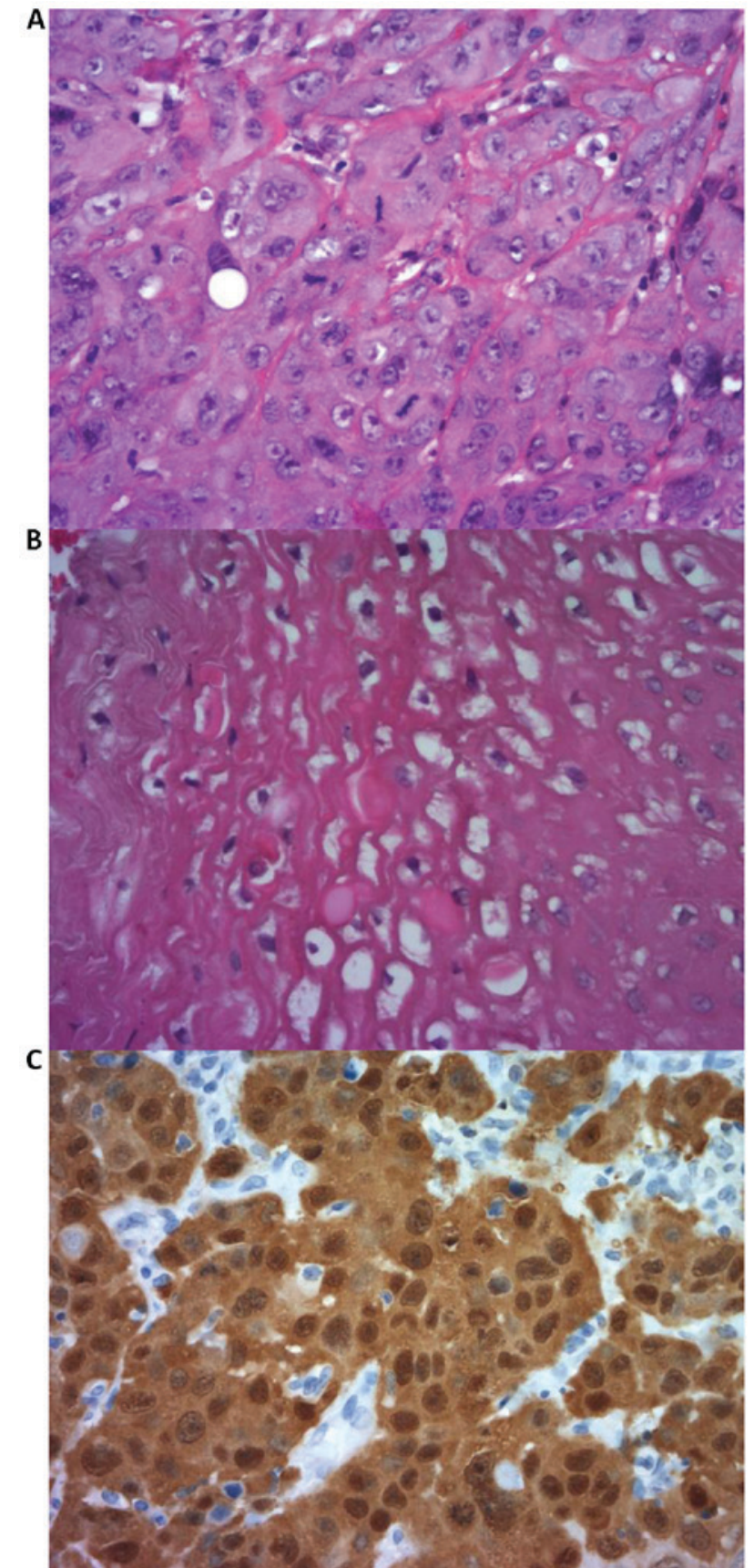

Figure 1. Histological hematoxylin and eosin and IHC analysis of oral tongue squamous cell carcinoma. (A) Sheets of moderate-poorly differentiated squamous cell carcinoma demonstrating pleomorphic vesicular nuclei with numerous mitotic figures and scant cytoplasm (magnification, x400). (B) Koilocytotic atypia characterized by vacuolated cytoplasm and dense cytoplasmic membranes (magnification, x400). (C) IHC staining for p16 demonstrates strong nuclear and cytoplasmic staining in $>70 \%$ of malignant cells. IHC, immunohistochemical.

Inc., Waltham, MA, USA). Immunostaining was mechanically performed on the LabVision ${ }^{\mathrm{TM}}$ Autostainer using the CINtec ${ }^{\circledR}$ p16 ${ }^{\text {INK4a }}$ detection system (REF 9517; Roche Diagnostics, Tucson AZ, USA). Staining was performed according to the manufacturer's protocol, with the exception that following the $30 \mathrm{~min}$ room temperature incubation with primary antibody the slides was stringently washed for 5 min in TBS containing $0.1 \%$ Tween 20 . The kit included the ready-to-use $\mathrm{CINtec}{ }^{\circledR}$ monoclonal mouse anti-Human $\mathrm{p} 16^{\mathrm{INK} 4 \mathrm{a}}$ antibody (Clone E6H4) and the negative control monoclonal mouse anti-rat oxytocin-related neurophysin. The slides were counterstained for 1 min with 1:5 dilution of Ready-to-Use Mayer's hematoxylin (Thermo Fisher Scientific, Inc., cat. no., TA-060-MH), dehydrated by sequential $1 \mathrm{~min}$ incubations in $95 \%$ ethanol, two rounds of $100 \%$ ethanol and 2 rounds of $100 \%$ xylene (Thermo Fisher Scientific, Inc.) and coverslipped using Cytoseal XYL (Thermo Fisher Scientific, Inc. cat. no., 9312-4). Images were captured using a Nikon EclipAcse E400 (Nikon Corporation, Tokyo, Japan) equipped with a Motic 5 MP digital webcam (Motic Instruments INC, Canada). Hematoxylin and eosin (H\&E) staining was performed using Hematoxylin 7211 and Eosin-Y Alcoholic kit (Thermo Fisher Scientific, Inc.).

p16 positive staining was concluded based on strong diffuse nuclear and cytoplasmic staining in $>70 \%$ of malignant cells. No staining, faint diffuse nuclear and cytoplasmic staining, small foci of staining or staining in non-malignant cells were considered negative results. The positive control was a tonsil cancer biopsy previously determined to be p16 positive as part of the standard clinical laboratory procedure.

Lynch Syndrome protein immunohistochemistry (IHC). Antigen retrieval, processing and staining methods, and image capture were conducted as previously described for p16 IHC. Antibodies for the four proteins were as follows: OAAF03051 MSH2, ARP41348P050 MSH6 antibody N-terminal region 100 and OAAF04086 PMS2 antibody (Aviva Systems Biology, San Diego CA, USA) and BDB550838 MLH-1 (BD Biosciences, Franklin Lakes NJ, USA).

As these mutations tend to be germline, all cells from individuals who do not have a mutation in one of these genes (histologically normal or tumor cells) should demonstrate evidence of the protein. The patterns of normal cellular expression of these proteins are as follows: MLH1 exhibits general nuclear expression with exclusion from nucleoli; MSH2 exhibits ubiquitous nuclear expression with exclusion from nucleoli and may be detected in vesicles; MSH6 exhibits nuclear expression with exclusion from nucleoli, and additional lower cytoplasmic expression in certain tissues (malignant cells for example); and normal tissues generally demonstrate weak to moderate nuclear positivity for PMS2, again with exclusion from nucleoli (12).

\section{Results}

Confirmation of OTSCC and the presence of histopathological and immunohistochemical hallmarks of HPV. Archival OTSCC tissue was processed for standard histopathological analysis using H\&E staining (Fig. 1A). The original diagnosis of moderate to well-differentiated OTSCC was confirmed, as was the presence of koilocytotic atypia (Fig. 1B), generally accepted as being pathognomonic for the presence of actively replicating HPV. The presence of HPV was additionally explored through use of the clinical lab-based methodology of IHC staining for elevated cellular p16, a surrogate marker for actively replicating HPV. Diffuse strong nuclear and cytoplasmic staining was observed in $>70 \%$ of malignant cells (Fig. 1C), which meets the diagnostic criteria for HPV infection 

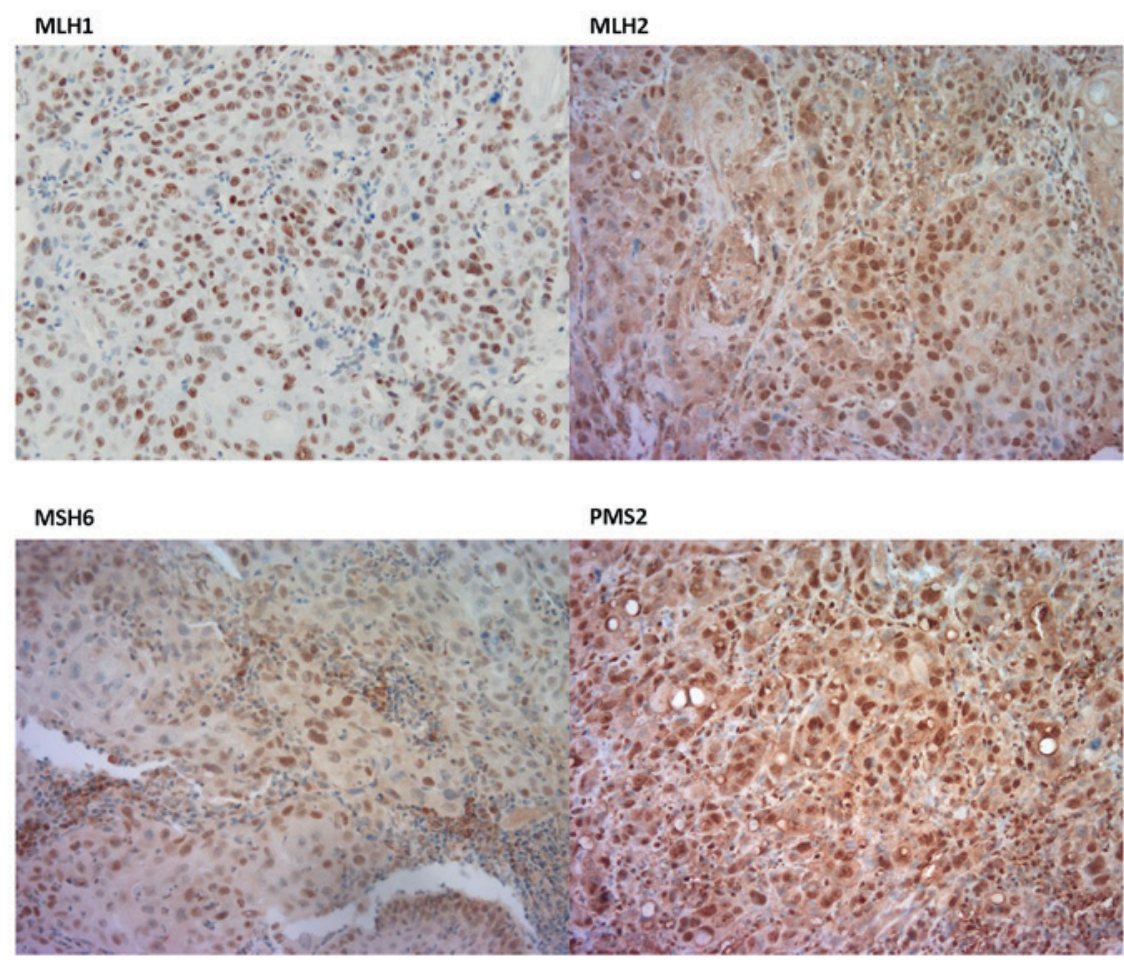

Figure 2. Immunohistochemical staining of oral tongue squamous cell carcinoma with antibodies for MLH1, MLH2, MSH6 and PMS2, the four proteins evaluated in the determination of Lynch syndrome (magnification, x200). Staining intensity and cellular patterns are normal. MLH, MutL homolog; MSH6, MutS homolog 6; PMS2, PMS1 homolog 2.

Lynch Syndrome protein immunohistochemistry. Lynch Syndrome is a syndrome in which a protein involved in DNA mismatch repair is not expressed due to a gene mutation. The four most common proteins associated with this syndrome are MLH1, MLH2, MSH6 and PMS2. IHC staining demonstrated normal expression levels and patterns for all four of these proteins (Fig. 2), concluding that the patient was not affected by Lynch Syndrome.

\section{Discussion}

The present study provides an illustration of a case of OTSCC in a young Caucasian adult, albeit one of only 21 years old. The causality, risk factors and association with HPV infection are unknown. A previous study examined a case in a patient as young as 19 years of age which is consistent with current trends (13). This referenced case as well as the case in the present study, demonstrate an emerging population of young women at increasing risk of tongue cancer.

Tobacco and alcohol use have been well-documented as risk factors for OTSCC. This fact is additionally confirmed by the decreasing trends in OTSCC, which is congruent with decreasing tobacco and alcohol use. However, the increasing incidence of OTSCC in young adults has indicated that there are emerging factors separate from tobacco and alcohol use. A unique feature of OTSCC in young patients has been the relative lack of these established risk factors. Previous studies and case reports continue to highlight this novel trend of the disease in young adults $(1,2,5,7)$. These patients exhibit insignificant contact with the known predisposing factors to oral tongue cancer. Even in patients $<30$ years old with a history of tobacco or alcohol use, the duration of exposure has been demonstrated to be insufficient for the development of malignancy (5). The patient in the present study did not report any significant risk factors, raising suspicions of the tongue cancer being HPV-associated. With the significant family history of malignancies, Lynch Syndrome was also considered.

Evidence for HPV included the initial suggestion on the pathology report of koilocytotic atypia and the confirmed strong p16 staining. The possibility of an underlying genetic risk that may have resulted in enhanced aggressive tongue cancer was also explored; however, the four Lynch Syndrome proteins were all detected at normal levels with normal intracellular distribution patterns. We hypothesized that there was something unique about the HPV genotype in this particularly aggressively growing tumor. However, it was not possible to purify DNA of a quality that would allow extensive evaluation by PCR or sequencing, and so the virus could not be genotyped (data not shown).

The HPV serotypes usually implicated in head and neck cancer are 16 and 18 (14). The prevalence of HPV16 had been estimated at 20-35\%, with the highest incidence of HPV infection noted in non-Hispanic Caucasian patients in the United States (14). Though young adult patients have been identified to present with similar symptoms to older adults, they tend to exhibit shorter symptom duration prior to diagnosis, and are more likely to be diagnosed at an advanced stage owing to the higher rate of regional involvement (5). OTSCC is increasingly being regarded as a biologically different entity compared with cancer affecting other oral sites. It is more aggressive and generally associated with a higher rate of metastasis (15). The patient of the present study, who already exhibited lymph node involvement with the short duration of the symptoms, exemplified this situation. 
The prognosis of OTSCC in young adults is uncertain. Certain studies have suggested higher disease recurrence and poor prognosis in young adults, while others have identified similar outcomes in this patient group as compared with in older adults: Pitman et al (16) demonstrated that with equivalent treatment, outcomes for young patients are analogous to those of older patients with OTSCC. Goepfert et al (17) and Vargas et al (18) observed that OTSCC in young females was not associated with worse outcomes compared with a matched cohort of other patients. This has suggested that age may be an independent prognostic factor for survival, as demonstrated by Garavello et al (19). The patient of the present study received a combination of chemotherapy and radiation and did not demonstrate any recurrence of disease during follow up. The 5-year relative survival demonstrated by Bello et al (15) indicated an overall higher survival rate among younger adults aged 20-44 years for OTSCC (64\%) compared with adults $>45$ years $(51 \%)$.

Oral tongue cancer incidence, presentation and causative factors, treatment protocol, survival and prognosis, particularly in young adults, continue to evolve and necessitate future studies.

\section{Acknowledgements}

The authors would like to thank Ms. Faye Jornadal, (Nashville General Hospital at Meharry, Nashville, TN, USA), for facilitating the acquisition of medical record information. The Meharry Office of Scientific Editing and Publications (grant no. NIH S21MD000104) provided formatting assistance.

\section{References}

1. Li R, Koch WM, Fakhry C and Gourin CG: Distinct epidemiologic characteristics of oral tongue cancer patients. Otolaryngol Head Neck Surg 148: 792-796, 2013.

2. Patel SC, Carpenter WR, Tyree S, Couch ME, Weissler M, Hackman T, Hayes DN, Shores C and Chera BS: Increasing incidence of oral tongue squamous cell carcinoma in young white women, age 18 to 44 years. J Clin Oncol 29: 1488-1494, 2011.

3. American Cancer Society. Cancer Facts \& Figures 2012. Atlanta, American Cancer Society, 2012.

4. Surveillance, Epidemiology and End Results (SEER) Program (www.seer.cancer.gov) SEER*Stat Database: Incidence-SEER 9 Regs Research Data, Nov 2015 Sub (1973-2013) <Katrina/Rita Population Adjustment $>$-Linked To County Attributes-Total U.S., 1969-2014 Counties, National Cancer Institute, DCCPS, Surveillance Research Program, Surveillance Systems Branch, released April 2016, based on the November, 2015 submission.
5. Llewellyn CD, Linklater K, Bell J, Johnson NW and Warnakulasuriya S: An analysis of risk factors for oral cancer in young people: A case-control study. Oral Oncol 40: 304-313, 2004.

6. Anaya-Saavedra G, Ramírez-Amador V, Irigoyen-Camacho ME, García-Cuellar CM, Guido-Jiménez M, Méndez-Martínez R and García-Carrancá A: High association of human papillomavirus infection with oral cancer: A case-control study. Arch Med Res 39: 189-197, 2008

7. Koch WM, Lango M, Sewell D, Zahurak M and Sidransky D: Head and neck cancer in nonsmokers: A distinct clinical and molecular entity. Laryngoscope 109: 1544-1551, 1999.

8. Siebers TJ, Merkx MA, Slootweg PJ, Melchers WJ, van Cleef P and de Wilde PC: No high-risk HPV detected in SCC of the oral tongue in the absolute absence of tobacco and alcohol-a case study of seven patients. Oral Maxillofac Surg 12: 185-188, 2008.

9. Marur S, D'Souza G, Westra WH and Forastiere AA: HPV-associated head and neck cancer: A virus-related cancer epidemic. Lancet Oncol 11: 781-789, 2010.

10. TNM Classification of Malignant Tumours, 6th edition. Sobin LH and Wittekind Ch (eds.). John Wiley \& Sons, Hoboken, New Jersey USA, pp22-263, 2002.

11. Backes FJ and Cohn DE: Lynch Syndrome. Clin Obstet and Gynecol 54: 199-214, 2011.

12. Shia J: Immunohistochemistry versus microsatellite instability testing for screening colorectal cancer patients at risk for hereditary nonpolyposis colorectal cancer syndrome. Part I. The utility of immunohistochemistry. J Mol Diagn 10: 293-300, 2008.

13. Randhawa T, Shameena P, Sudha S and Nair R: Squamous cell carcinoma of tongue in a 19-year-old female. Indian J Cancer 45: 128-130, 2008.

14. Gillison ML: Human papillomavirus-associated head and neck cancer is a distinct epidemiologic, clinical, and molecular entity. Semin Oncol 31: 744-754, 2004.

15. Bello IO, Soini Y and Salo T: Prognostic evaluation of oral tongue cancer: Means, markers and perspectives (II). Oral Oncol 46: 636-643, 2010.

16. Pitman KT, Johnson JT, Wagner RL and Myers EN: Cancer of the tongue in patients less than forty. Head Neck 22: 297-302, 2000.

17. Goepfert RP, Kezirian EJ and Wang SJ: Oral tongue squamous cell carcinoma in young women: A matched comparison-do outcomes justify treatment intensity? ISRN Otolaryngol 2014: $1-6,2014$

18. Vargas H, Pitman KT, Johnson JT and Galati LT: More aggressive behavior of squamous cell carcinoma of the anterior tongue in young women. Laryngoscope 110: 1623-1626, 2000.

19. Garavello W, Spreafico R and Gaini RM: Oral tongue cancer in young patients: A matched analysis. Oral Oncol 43: 894-897, 2007.

(c) (1) () $९$ This work is licensed under a Creative Commons (c) NO NO Attribution-NonCommercial-NoDerivatives 4.0 International (CC BY-NC-ND 4.0) License. 